\title{
Paper Fuzzy Clustering with Multi-Constraint QoS Service Routing in Wireless Sensor Networks
}

\author{
Jayashree Agarkhed, Vijayalaxmi Kadrolli, and Siddarama R. Patil \\ P.D.A. College of Engineering, Kalaburagi, India
}

https://doi.org/10.26636/jtit.2019.127818

\begin{abstract}
This paper presents a fuzzy logic-based, service differentiated, QoS aware routing protocol (FMSR) offering multipath routing for WSNs, with the purpose of providing a service differentiated path meant for communication between nodes, based on actual requirements. The proposed protocol initially forms a cluster by fuzzy c-means. Next, the building of a routing follows, so as to establish multiple paths between nodes through the modified QoS k-nearest neighborhood, based on different QoS constraints and on optimum shortest paths. If one node in the path fails due to lack of residual energy, bandwidth, packet loss, delay, an alternate path leading through another neighborhood node is selected for communication. Simulation results show that the proposed protocol performs better in terms of packet delivery ratio, delay, packet drop ratio and throughput compared to other existing routing protocols.
\end{abstract}

Keywords—fuzzy logic, QoS, routing, WSN.

\section{Introduction}

Energy efficient routing is the main objective of wireless sensor networks (WSNs). In WSNs, sensor nodes collaborate with each other by communicating with neighboring nodes. They also perform basic computations based on the data collected and complete different tasks, such as neighborhood node discovery, smart sensing and optimal efficient routing - at every layer. The routing protocols are classified in terms of QoS aware protocols and performance [1]-[3]. In order to provide QoS in the applications, in most of the cases fuzzy logic-based selection of cluster head is used in the course of the routing process, which provides a non-probabilistic approach with two fuzzy variables: one is base station distance and the other is residual energy of the sensor nodes.

Multi-hop communication is used for the selection of cluster head $(\mathrm{CH})$. This has the authority to communicate with other CHs and with BS. Various methods are used to identify the next forwarding node. The selection of nodes is based on different techniques, such as fuzzy logic, neuro fuzzy and the mobility of nodes [4].

Flooding is also used to set up possible routes to destinations which rely on bandwidth, node energy or link quality. As a consequence, these strategies may lead to unneces- sary message transmissions, network jamming, longer delays and loss of packets. To avoid these problems, it is essential to come across the optimal path between nodes using the existing resources in the network. However, attempting to choose a route that satisfies many constraints may result in conflicts and the process may be complicated. Therefore, it is recommended to deploy multi-metrics in WSNs, with path and packet communication based on differentiated services [5].

An effective, optimal, multipath, service-differentiated routing protocol, known as the fuzzy-based service-differentiated QoS-aware routing protocol (FMSR), is proposed, which initially forms a cluster by fuzzy c-means and uses multiple metrics, such as link bandwidth, residual energy, packet loss and delay to choose the neighborhood nodes. Multiple paths are subsequently established between the source and the destination, leading through these neighborhood nodes, by means of the k-nearest neighborhood method, thus forming an optimal route for differentiated services. If a neighborhood node along the path fails due to lack of bandwidth or energy, an alternative path is established.

In Section 1 an introduction to the paper is presented. Section 2 contains a short survey of the existing routing protocols. The plan of the proposed work is described in Section 3. Finally, performance, simulation results and conclusions are summarized in Sections 4 and 5.

\section{Related Work}

Several studies have been conducted to attempt, with varying degrees of success, to address the problem of energyefficient, delay-constrained routing in WSNs and multiple metrics are used for routing, considering link rate and packet loss, i.e. [6].

Soft computing methods have been truly helpful in a variety of areas and have shown capable outputs. Novel clustering algorithms are based on the fuzzy c-means concept, where the selection of the cluster head is based on its proximity to the middle of the cluster and to the node having the highest residual energy. Non-cluster head nodes broadcast sensed data to the cluster head perform data aggregation and transmit data straight to the base station [7], [8]. 
Fuzzy logic control, known as BOKHARI-SEPFL, based on distance of nodes form the base station, density of nodes and energy level, as well as traditional threshold values are used to enhance the process of cluster head election to improve the lifetime and throughput of the WSN [9].

An energy efficient adaptive routing is proposed in the form of a fuzzy-based clustering protocol that makes use of the direct transmission technique, depending upon the criticality and the location of sensor nodes [10].

Mamdani's fuzzy inference system is used to identify the ability of a sensor node to become a group cluster head, depending on the distance of the input parameter and the energy of the sensing nodes. The heuristic search algorithm is used to find the minimum path length from the source to the receiving node. The aggregated data packets are routed from the originating $\mathrm{CH}$ to the receiving node along the selected route [11].

Energy-aware routing protocols have been proposed for WSNs. Most of them are energy savers and there is not much focus on energy balancing. Though, the lifetime of WSNs severely depend energy use; so, energy management is a necessary job to be considered [11], [12]. The energy aware routing protocol - FEAR which balances energy and energy saving, is considered. It shows an appropriate tradeoff between the saving of energy and energy balancing by a fuzzy set scheme. Based on examination of energy expenditure for the data transceiver, a single-hop forwarding system is proved to provide less energy than multi-hop forwarding [13], [14].

The main advantage of the fuzzy logic control-based QoS management (FLC-QM) method consists in changing traffic load. It utilizes a fuzzy logic controller, relying on the source sensor node to get the sampling period and the deadline miss ratio for the transmission of data from the sensor to the actuator [15], [16].

A protocol has been introduced that exchanges roles between regular nodes and cluster heads in a round robin manner, following the token ring methodology. The equidistribution of cluster head burden over all sensors in the same cluster reduces the need of expensive periodic re-clustering. The domain memberships of edge sensors are handled through fuzzy logic, based on the residual energy [17].

The rumor routing algorithm in WSNs allows the query source to distribute the query to identify a source which helps get a timely query message, but energy efficiency is improved by relying on the hierarchical clustering formation method, while the fuzzy logic method is a used to increase network efficiency [18].

Intelligent multipath routing has been used, which uses fuzzy stochastic multipath routing (FSMR) for providing hop count, battery power and signal strength. Nodes are stochastically forwarded with path selection, which results in automatic load balancing and fault tolerance [19], [20]. Geographic opportunistic routing (GOR) used to provide QoS with end-to-end reliability and delay restrictions in WSNs, for different opportunities, has been proposed to ensure multi control service quality in WSNs - a problem that may be formulated as one involving multi-objective optimization, i.e. selection and prioritization of a set of candidates for efficient forwarding. The solution is suitable for WSN in terms of efficiency energy, latency and temporal complexity [21].

An adaptive multi-constraint multipath routing protocol which minimizes loss rate, energy consumption and delay between clusters, based on a weighted cost function and on such parameters as loss rate, residual energy and delay, is presented in [18]. An approach to calculate approximately probabilistic timeliness guarantees end-to-end communication delivery delays in WSNs and is used at run-time to build a metric which estimates the probability density function of the end-to-end latency of a path [22]. In fuzzy stochastic multipath routing (FSMR), multiple metrics are used to determine hop count, battery power, signal strength and fuzzy logic is used to offer multiple optimal paths [23]. A novel relative mobility metric for mobile ad hoc networks (MANETs), which is based on power level ratios changing at every node due to consecutive receptions from its neighbors, is addressed in [24].

\section{System Architecture and Methodology}

The proposed work identifies multiple paths between nodes, leading through candidate nodes, based on different QoS constraints. The proposed architecture is shown in Fig. 1. It comprises 3 parts:

- cluster formation by using fuzzy c-mean,

- optimal shortest routing by using k-nearest neighborhood method,

- providing service differentiations based on service requirements.

QoS nodes based on different constraints are known as candidate nodes and are chosen from the $\mathrm{CH}$ set. These nodes are chosen based on residual energy, bandwidth, packet loss and delay. The distance of the $\mathrm{CH}$ from the sink is essential for energy efficiency and is crucial for balancing energy spending and network lifetime. Hence, the formation of clusters between sensor nodes is given priority. The fuzzy c-means clustering approach is used in cluster formation to determine the set of $k$ clusters in $d$-dimensional space. In the network structure, each sensor node has maintained a routing table with neighbor node distance, residual energy, bandwidth and packet loss between nodes. Before starting any operation, each sensor node initializes each sensor by exchanging the routing table. After initiating, each node starts the transmission phase. Before the transmission, it checks the required bandwidth, delay, residual energy and packet loss by comparing it with the threshold value set. The routing path is a set of applicant nodes based on different QoS parameters. If any QoS applicant 


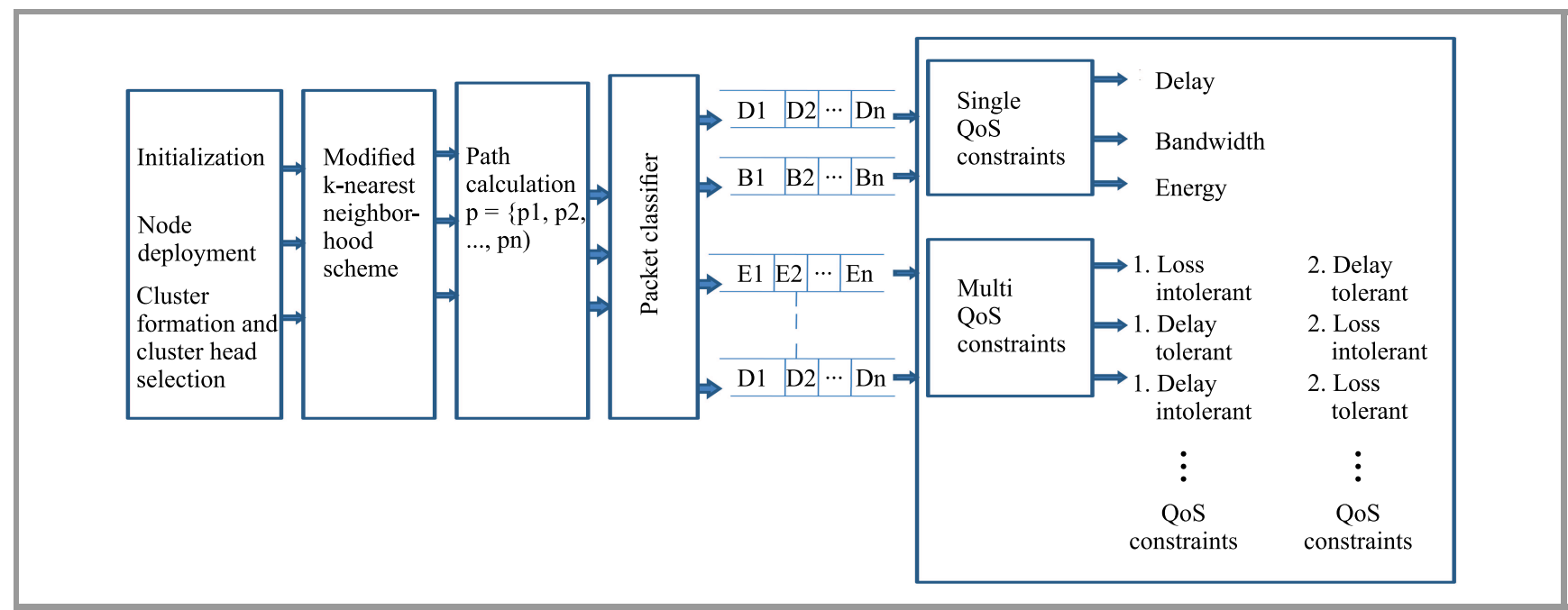

Fig. 1. System architecture.

node in the path fails because it does not meet the requirements related to bandwidth, residual energy, packet loss, an alternate path is established.

In the initialization phase, every sensor node telecasts a hello message to neighboring nodes, keeping in mind that the end goal is to have enough high quality information. Every sensor node maintains and updates a table with neighboring nodes during this stage. The table contains a list of the sensor node's neighboring nodes. While establishing each path, the sensor node sends a hello packet to another node. If the parameters are met, then the path is established (Fig. 2).

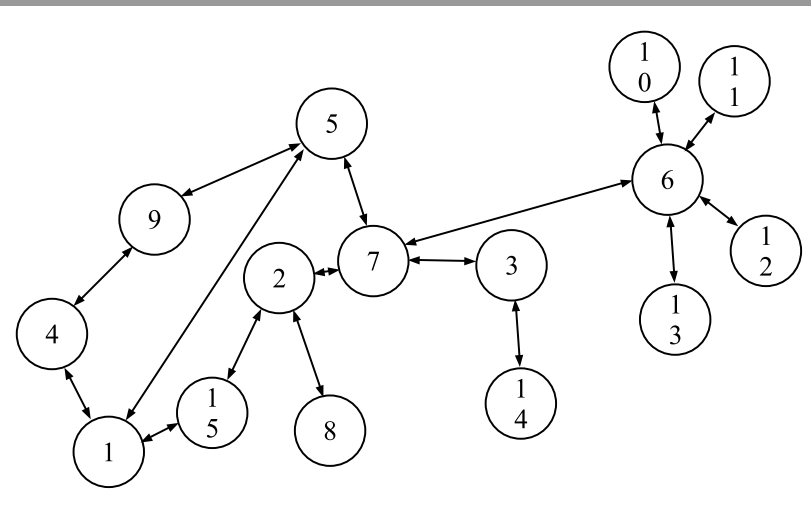

Fig. 2. Route initialization phase.

Next, cluster formation proceeds and the fuzzy c-means clustering algorithm is applied. It uses the information about its members to select the right option. Then, cluster head formation is calculated and the cluster head is selected for each cluster.

In the neighborhood scheme, a modified QoS k-neighborhood algorithm is used. The distance between $\mathrm{CH}$ and sink is calculated by the Euclidian distance (Algorithm 1). This demonstrates how to route data information based

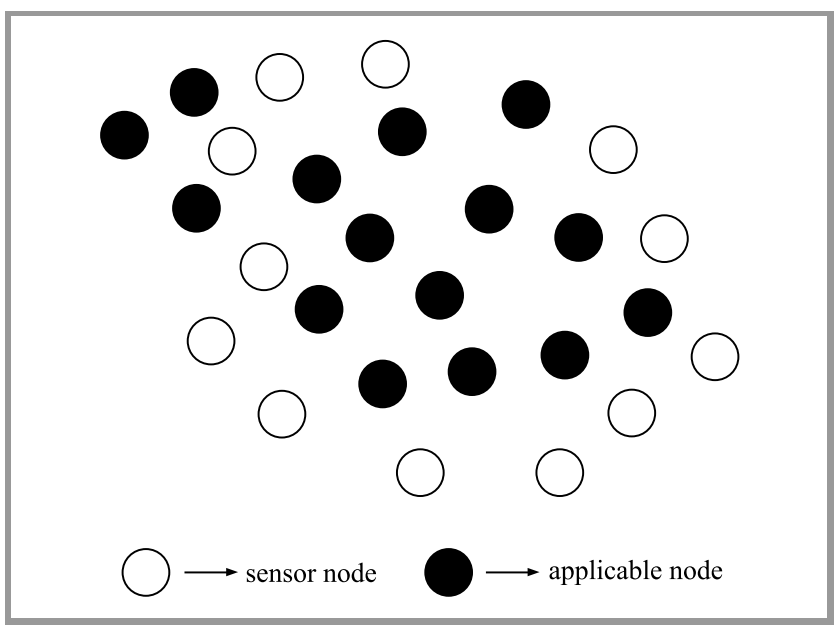

Fig. 3. Applicable cluster node selection.

on QoS parameters: residual energy, delay, bandwidth and packet loss. Within the network structure, each sensor node maintains a routing table that contains neighbor node distance, residual energy, bandwidth and packet loss experienced while communicating with other nodes, as shown in Fig. 3. Before commencing any operation, each censor node is initialized, i.e. it exchanges the routing table. After initiating the transmission phase, but before the transmission, it checks the minimum bandwidth, delay, residual energy and packet loss.

To validate the routing, four metrics are used:

- Bandwidth. When a node intends to transport data, it has to be conscious of the local bandwidth and the interference and transmission range of the neighboring nodes. Therefore, the node needs to monitor the channel and estimate local bandwidth (LBW) which depends on the idle-to-busy time ratio. Residual energy is computed by the node over a given period of time. 
- Packet loss rate (PLR). The packet loss rate is calculated as the ratio between the amount of data packets received and the total amount of data packets sent.

- Delay - is the time difference between the time when the packet was sent from source and he time when it arrives at the other node.

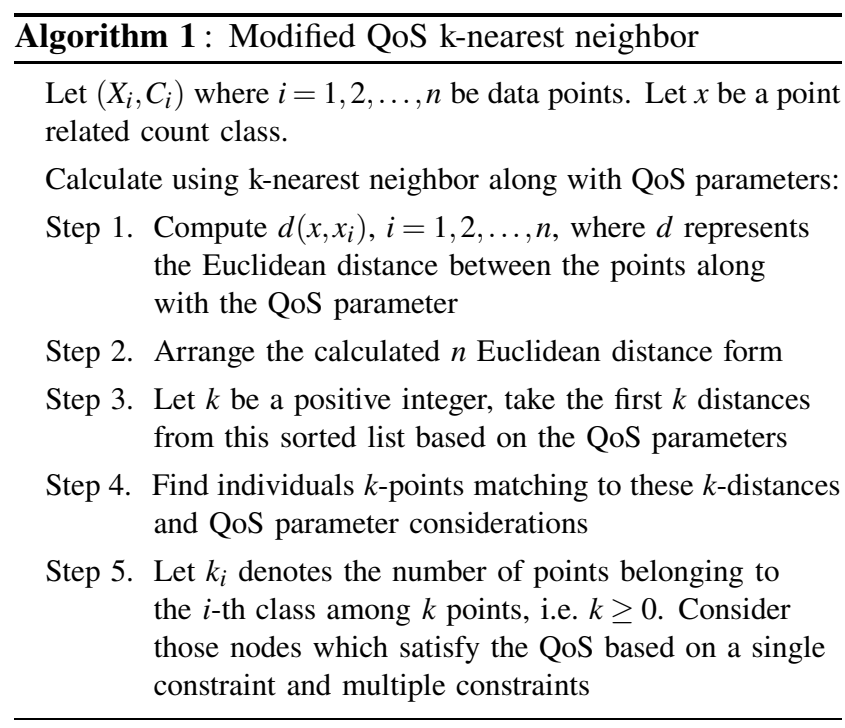

In the next step service differentiations are provided based on service requirements. Packet and path classifiers are determined as well (Fig. 4).

Packets are classified based on QoS constraints. If packets are delay sensitive, then they are sent to the delay sensitive path. If packets are bandwidth sensitive, then they are sent to the bandwidth sensitive path. If packets are energy sensitive, then they are sent to the energy sensitive path. The best available path matching the service type is considered for routing the data. The path with the lowest energy consumption, delay, bandwidth and packet loss is considered to be the optimal path.

\subsection{Path Discovery Phase}

When a source node intends to broadcast a data packet to a target node, it initiates the multipath routing detection process between the source and the destination. This initiates the routing process. The source node must first check its routing table to determine whether the routing table contains information. If a route is established, the source will make use of the route to send the data packet instantly, or else, the source node will broadcast a route request (RREQ) packet.

The state of $d$ node indicates whether the node is designated as a candidate node or a non-candidate node. When a node receives an RREQ packet, it will forward the packet to all its neighbors. When an intermediate node receives an RREQ packet, if it has previously received an RREQ packet with a similar series number and destination ID, it drops the unnecessary RREQ packet. When an intermediate node receives an RREQ packet for the first time, it updates its routing table with the source ID and destination ID and the previous hop node ID and its state and appends its state to the RREQ message in the node state field and analyzes the destination ID. When the destination node receives the RREQ message, it appends its state to the route reply (RREP) and unicasts the reply message on the reverse path toward the source. The destination performs this action for every RREQ it receives. At the same time, an intermediate node receives the RREP message, it appends its state to the message, updates its routing table and unicasts the RREP in the direction of the source using the formerly stored hop node information. The source chooses a path of applicable nodes to transmit the data packet.

\subsection{Data Transmission Phase}

Figure 5 shows the optimal path's source (thin line, path 3) and destination nodes, indicated by a square box. The ser-

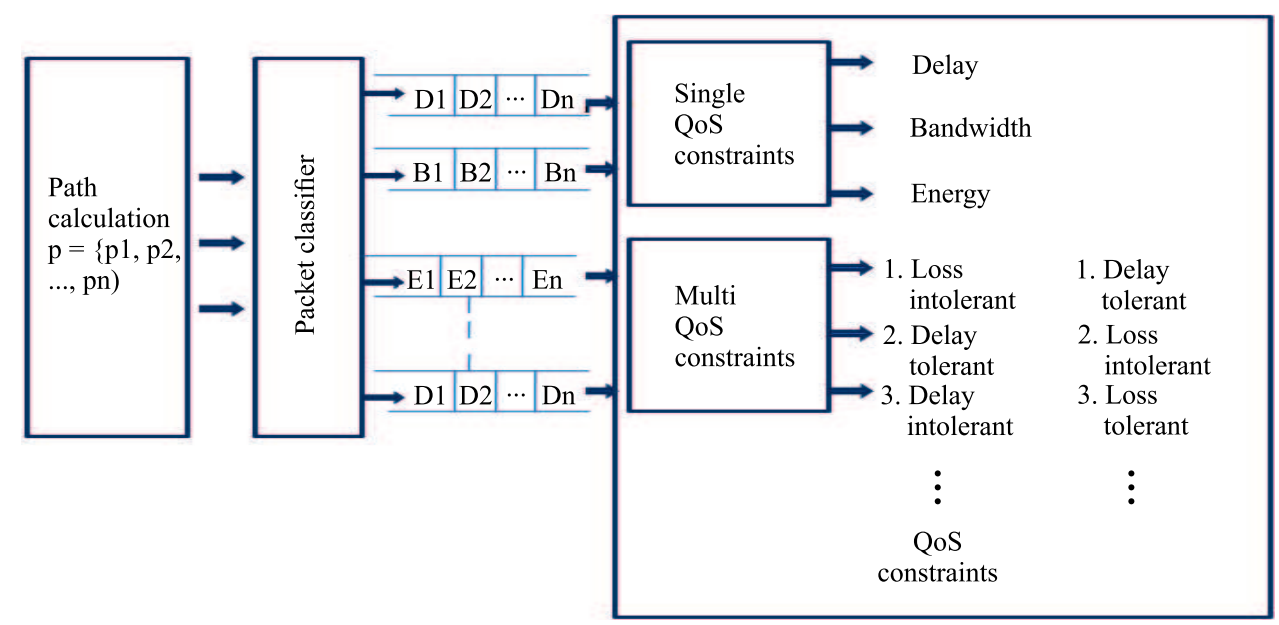

Fig. 4. Packet and path classifier. 


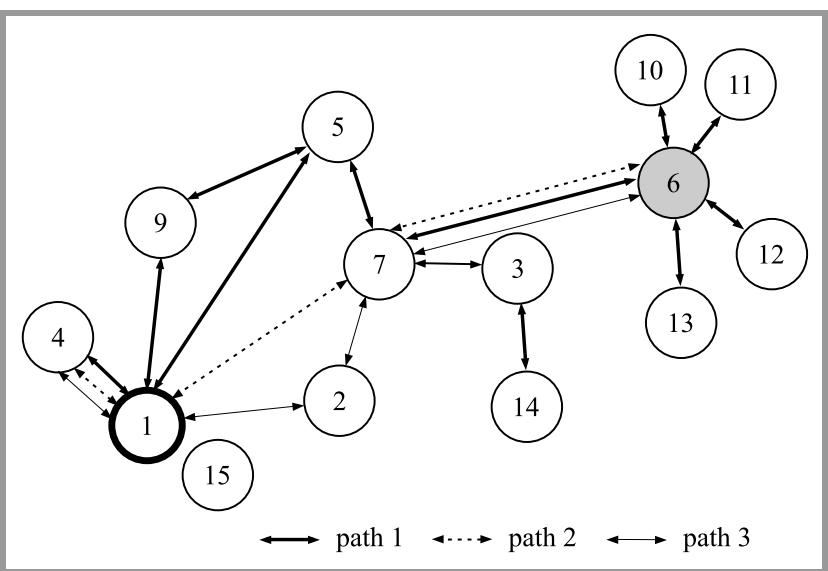

Fig. 5. Path discovery.

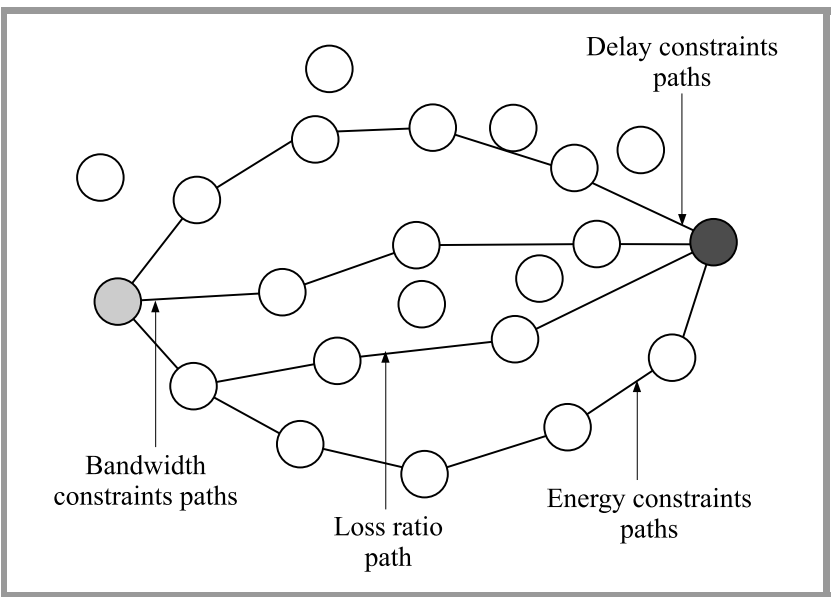

Fig. 6. Service differentiation paths.

vice differentiation paths are shown in Fig. 6. After receiving transferring of sense data it represented output results by the graph. For example, in the figure shown, node 6 wants to send data to node then it will find a destination node using multipath paths are For example in the figure, node 6 wants to send data to node then it will find a destination node using multipath paths are:

- 1.6-7-5-9-1 - in this path, the hop count is larger, it consumes more energy and node 9 is busier than in other paths,

- 2.6-7-1-4 - in this path the hop count is lower, less energy is consumed and BW is greater,

- 3.6-7-2-1-4 - in this path the hop count is higher, more energy is consumed and BW is lower compared to path 6 .

Checking all parameters, select the optimized path used to transmit data from the source to the destination.

\section{Simulation System and Parameters}

A comparison of the proposed protocol with existing protocols is performed using the NS-2 network simulator [25].
The simulation models use a network of 100 nodes in a $500 \times 500 \mathrm{~m}$ region, with the number of sensor nodes varying from 0 to 100 . The average of 10 runs has been performed for simulation purposes. Each and every node randomly selects a position and moves in the direction of that position. Once the node arrives at the position, it stays there for a predefined period of time. After that time, it selects a new position and repeats the process. The simulations run lasts for $200 \mathrm{~s}$.

The evaluation of the proposed protocol is based on relevant parameters - the number of packets dropped, delay, bandwidth and hop count.

\subsection{Comparative Analysis}

Figure 7 shows a comparison between the packet delivery ratio (PDR) in the proposed network and in the existing protocol [14], with varying times. As the time increases, the number of packets delivered increases in the existing protocol. The existing protocol's FSMR chooses the optimal route for transmission and finds an alternate path through the applicable nodes when the path breaks. When applicable nodes are selected, no other nodes are involved and they are sent to sleep mode. Consequently, it offers better PDR in terms of different QoS constraints.

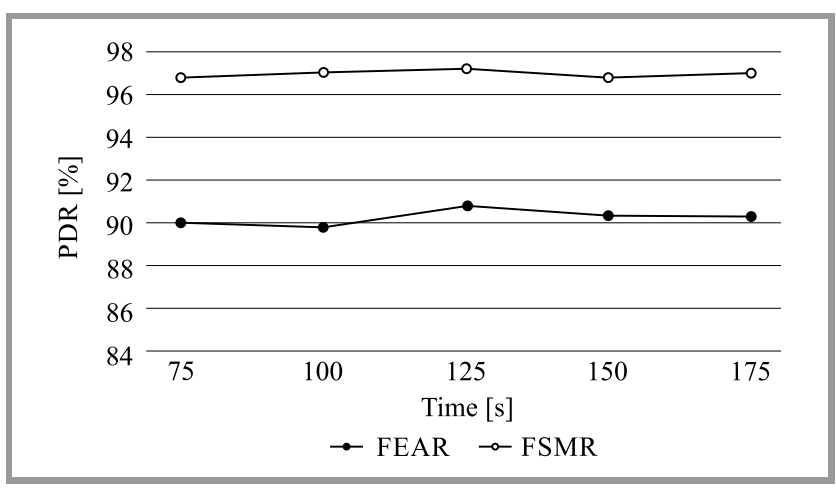

Fig. 7. PDR versus time.

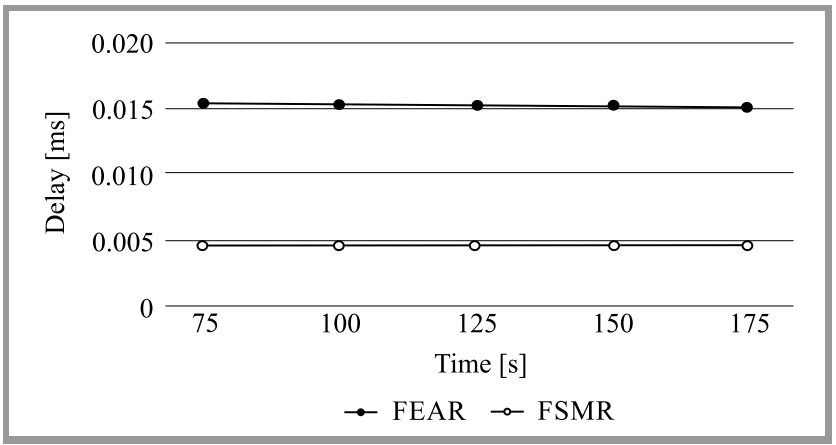

Fig. 8. Delay versus time.

Figure 8 shows that the delay in FSMR will be lower than in FEAR, because of the fact that applicable nodes have already considered the delay parameter, and if the link breaks, another applicable node will be chosen to estab- 
lish the route. FSMR offers also a better service based on QoS constraints, and a separate path with a specific delay is maintained. It checks whether the QoS delay parameter is satisfied or not for each link. It separates all available paths only by considering the most prominent delay, and selects one optimal path based on the delay constraint and the shortest path to transmit the packet.

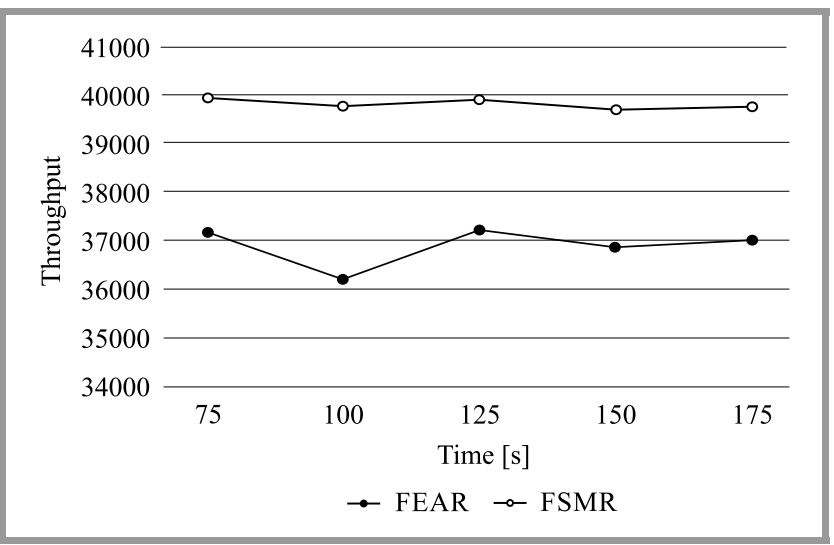

Fig. 9. Throughput versus time.

Figure 9 shows that the delay of FEAR increases as the time increases, because the longer time may deplete more energy. This may lead to packet loss, buffer overflow, degradation in throughput and frequent route breaks. Therefore, it results in a longer end-to-end delay. FSMR achieves the best path to transmit the data from the source to the designation before transmitting any packets, so the link selecting process has to be performed. The selected path should have a lower mean end-to-end delay.

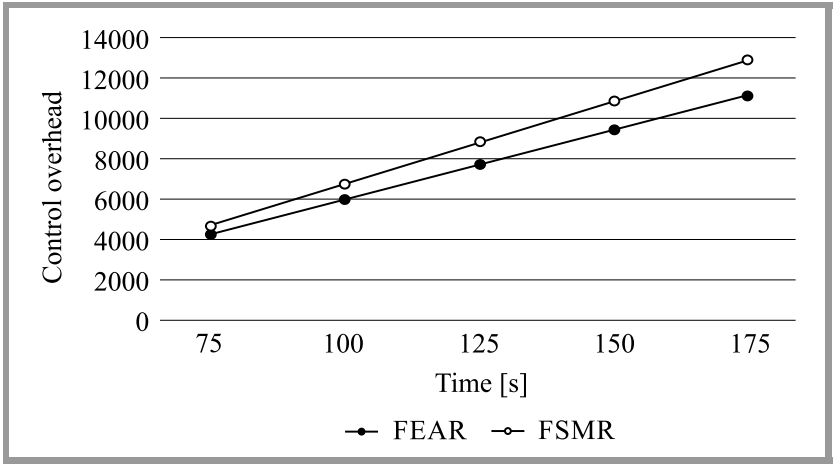

Fig. 10. Control overhead versus time.

Figure 10 shows the behavior of the proposed protocol according to the control overhead. It shows the packets that are sent and received with the use of a specific route. A different simulation time has been adopted here for each of the iterations, while the characteristics (initial power, node distribution and distance from sink) remain the same. The average of 10 simulation runs have been conducted to evaluate performance.

The overall control overhead is reduced for FSMR, because adopting fuzzy logic, and also by using the selected nodes only to establish the route for servicing the packets for particular services.

\section{Conclusion}

The purpose of the proposed protocol is to find the optimized path between nodes, using relevant nodes. The node selection process is performed using the modified QoS knearest neighborhood technique. Simulation performed in the NS2 simulator shows that the proposed technique reduces the delay, as well as increases the packet delivery ratio and throughput.

\section{References}

[1] S. Hasan, Z. Hussain, and R. K. Singh, "A survey of wireless sensor network", Int. J. of Emerg. Technol. and Adv. Engin., vol. 3, no. 3, pp. 487-492, 2013 [Online]. Available: https://ijetae.com/files/Volume3Issue3/IJETAE_0313_83.pdf

[2] M. Asif, S. Khan, R. Ahmad, M. Sohail, and D. Singh, "Quality of service of routing protocols in wireless sensor networks: A survey", IEEE Access, vol. 5, pp. 1846-1871, 2017 (doi: 10.1109/ACCESS.2017.2654356).

[3] V. Kadrolli and J. Agarkhed, "Soft computing routing techniques in wireless sensor network", in Proc. 2nd Int. Conf. on Adv. in Electric., Electron., Inform., Commun. and Bio-Inform. AEEICB 2016, Chennai, India, 2016, pp. 748-751 (doi: 10.1109/AEEICB.2016.7538395).

[4] S. Boukerche et al., "Routing protocols in ad hoc networks: A survey", Comp. Networks, vol. 55, no. 13, pp. 3032-3080, 2011 (doi: 10.1016/j.comnet.2011.05.010).

[5] F. Ahamad and R. Kumar, "Energy efficient region based clustering algorithm for WSN using fuzzy logic", in Proc. IEEE Int. Conf. on Recent Trends in Electron., Inform. \& Commun. Technol. RTEICT 2016, Bangalore, India, 2016, pp. 1020-1024 (doi: 10.1109/RTEICT.2016.7807984)

[6] T. A. Muthupandian, J. G. Eanoch, and H. Robinson Yesudhas, "A survey on techniques for selection of forwarding node in wireless sensor networks", Int. J. of Adv. in Comp. and Electron. Engin., vol. 2, no. 4, pp. 24-29, 2017.

[7] G. Santhi and A. Nachiappan, "Fuzzy-cost based multi constrained QoS routing with mobility MANETs", Egyptian Informat. J., vol. 13, no. 1, pp. 19-25 2012 (doi: 10.1016/j.eij.2011.12.001).

[8] C. Intanagonwiwat, R. Govindan, and D. Estrin, "Directed diffusion: A scalable and robust communication paradigm for sensor networks", in Proc. of the 6th Ann. Int. Conf. on Mobile Comput. and Network. MobiCom'00, Boston, MA, USA, 2000, pp. 56-67 (doi: 10.1145/345910.345920).

[9] A. Nanda and A. K. Rath, "Mamdani fuzzy inference based hierarchical cost effective routing (MFIHR) in WSNs", in Proc. IEEE 7th Int. Adv. Comput. Conf. IACC 2017, Hyderabad, India, 2017, pp. 7-401 (doi: 10.1109/IACC.2017.0089).

[10] K. Singh and R. K. Singh, "An energy efficient fuzzy based adaptive routing protocol for wireless body area network", in Proc. IEEE UP Section Conf. on Elec. Comp. and Electron. UPCON 2015, Allahabad, India, 2015, pp. 1-6 (doi: 10.1109/UPCON.2015.7456680).

[11] S. V. Mallapur and S. R. Patil, "Fuzzy logic-based stable multipath routing protocol for mobile ad hoc networks", in Proc. Ann. IEEE India Conf. INDICON 2014, Pune, India, 2014, pp. 1-6 (doi: 10.1109/INDICON.2014.7030670)

[12] M. U. Bokhari, "Bokhari-SEPFL routing protocol based on fuzzy logic for WSNs", in Proc. 5th Int. Conf. on Reliab., Infocom Technol. and Optimiz. ICRITO 2016 (Trends and Future Directions), Noida, India, 2016, pp. 38-43 (doi: 10.1109/ICRITO.2016.7784920). 
[13] S. Souiki, M. Hadjila, and M. Feham, "Fuzzy based clustering and energy efficient routing for underwater wireless sensor networks", Int. J. of Comp. Netw. \& Commun. (IJCNC), vol. 7, no. 2, pp. 33-44, 2015 (doi: 10.5121/ijcnc.2015.7203).

[14] E. Ahvar, A. Pourmoslemi, and M. J. Piran, "FEAR: A fuzzy-based energy-aware routing protocol for wireless sensor networks", arXiv preprint arXiv:1108.2777 [Online]. Available: https://arxiv.org/ftp/ arxiv/papers/1108/1108.2777.pdf

[15] H. Jiang, Y. Sun, R. Sun, and H. Xu, "Fuzzy-logic-based energy optimized routing for wireless sensor networks", Int. J. of Distrib. Sensor Netw., vol. 9, no. 8, 2013 (doi: 10.1155/2013/216561).

[16] M. Omari, H. Abdelkarim, and B. Salem, "Optimization of energy consumption based on genetic algorithms optimization and fuzzy classification", in Proc. 2nd World Symp. on Web Appl. and Network. WSWAN 2015, Sousse, Tunisia, 2015 (doi: 10.1109/WSWAN.2015.7210317).

[17] F. Xia, W. Zhao, Y. Sun, and Y. C. Tian, "Fuzzy logic control based QoS management in wireless sensor/actuator networks", Sensors, vol. 7, no. 12, pp. 3179-3191, 2007 (doi: 10.3390/s7123179).

[18] R. V. Dharaskare and M. M. Goswami, "Intelligent multipath routing protocol for mobile ad hoc network", Int. J. of Comp. Sci. and Appl., vol. 2, 2009, pp. 135-145.

[19] L. Cheng et al., "QoS aware geographic opportunistic routing in wireless sensor networks", IEEE Trans. on Parallel and Distrib. Syst., vol. 25, no. 7, pp. 1864-1875 (doi: 10.1109/TPDS.2013.240).

[20] J. Agrakhed, G. S. Biradar, and V. D. Mytri, "Adaptiv multi constraint multipath routing protocol in wireless multimedia sensor network", in Proc. Int. Conf. on Comput. Sci., Phagwara, India, 2012, pp. 326-331 (doi: 10.1109/ICCS.2012.9).

[21] R. S. Oliver "Estimation of the probability density function of endto-end delays in wireless sensor networks", Tech. Rep., Technische Universität Kaiserslautern, Kaiserslauter, Germany, Jan. 2009 [Online]. Available: https://rts.eit.uni-kl.de/fileadmin/ publication_files/TR09_serna_oliver.pdf

[22] T.-S. Su, C.-H. Lin, and W.-S. Hsieh, "A novel QoS-aware routing for ad hoc networks", in Proc. of the 9th Joint Int. Conf. on Inform. Sci. JCIS 2006, Kaohsiung, Taiwan, China, 2006 (doi: 10.2991/jcis.2006.117).

[23] V. Rashiwal, S. Verma, and S. K. Bajpai, "QoS based power aware routing in MANETs", Int. J. of Comp. Theory and Engin., vol. 1, no. 1, pp. 49-54 (doi: 10.7763/IJCTE.2009.V1.8)

[24] P. Basu, N. Khan, and T. D. C. Little, "A mobility based metric for clustering in mobile ad hoc networks", in Proc. 21st Int. Conf. on Distrib. Comput. Syst. Worksh. ICDCS 2001, Mesa, AZ, USA, 2001, pp. 413-418 (doi: 10.1109/CDCS.2001.918738).

[25] Network Simulator [Online]. Available: https://www.isi.edu/nsnam/ns

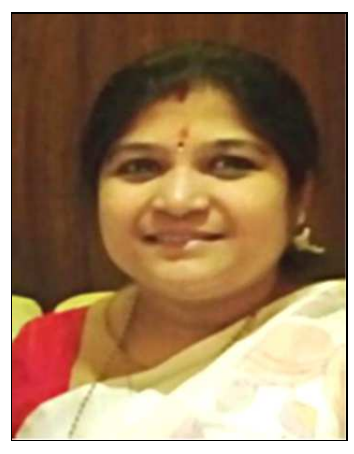

Jayashree V. Agarkhed is currently working as professor at the Computer Science and Engineering Department of the Poojya Doddappa Appa College of Engineering, Gulbarga, Karnataka, India. She received her BE degree from Gulbarga University, Gulbarga, Karnataka, India, as well as M.Tech. and Ph.D. degrees from Visveshwaraya Technological University, Belagavi, Karnataka, India in 1999, 2003 and 2013, respectively. She supervised numerous BE and M.Tech. projects and guided more than $8 \mathrm{Ph}$.D. students. She is a lifetime member of the Indian
Society for Technical Education, an India member of the Institute of Electrical and Electronics Engineers, a member of the Institute of Electronics and Telecommunication Engineers, India, and a member of the Institute of Engineers, India. Her research interests are in the area of wireless networking with QoS provisioning, as well as scheduling and routing algorithm design in sensor networks, Ad Hoc Networks and cloud computing. She has published more than 125 scientific articles in top-tier journals and conferences. She has also published 2 books. She has chaired various international conferences and is the reviewer for various National and International journals and Conferences. She is the Member of Board of Studies (BOS) and Board of Examiners (BOE) of computer science and engineering department and also the member of IEEE, ACM-W and IEI, life member of CSI and fellow member of ISTE and IETE.

(iD) https://orcid.org/0000-0003-3365-6498

E-mail: jayashreeptl@yahoo.com

Department of CSE

P.D.A College of Engineering

Kalaburagi, India

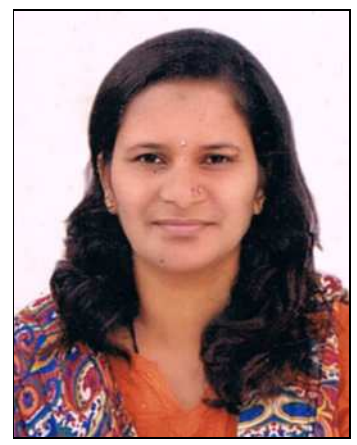

Vijayalaxmi Kadrolli is currently a Research Scholar at the Computer Science and Engineering Department at Poojya Doddappa Appa (PDA) College of Engineering, Kalaburagi, Karnataka, India, an autonomous institute affiliated to Visvesvaraya Technological University (VTU), Belagavi, Karnataka, India. She obtained her M.Tech. in Computer Science and Engineering from the P.D.A. College of Engineering, VTU, in 2004. Her main research areas are in wireless sensor networks, artificial intelligence and soft computing.

(D) https://orcid.org/0000-0003-4349-519X

E-mail: udachanv@gmail.com

Research Scholar

Department of CSE

P.D.A. College of Engineering

Kalaburagi, India

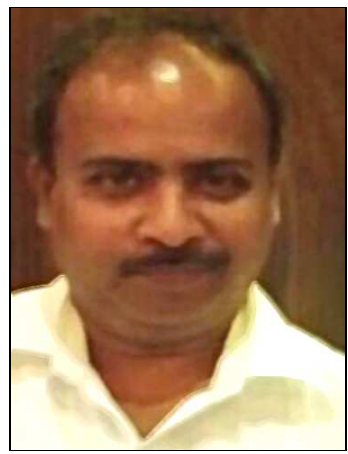

Siddarama R. Patil received his B.E. degree in Electronics and Communication Engineering from Gulbarga University, Gulbarga, Karnataka, India, M.Tech. in Telecommunication Engineering and Ph.D. from the Indian Institute of Technology (IIT), Khargpur, India in 1990, 1999 and 2009, respectively. Currently, he is 
a Professor and Dean Academics at Poojya Doddapa Appa College of Engineering, Kalaburgi, Karnataka, India. He has published more than 50 research papers in top-tier journals and conferences, including Springer journal, IEEE conference proceedings and Springer Book chapters. He has guided many BE and M.Tech. Projects and guiding more than $8 \mathrm{Ph} . \mathrm{D}$. students. He is a life member of Indian Society for Technical Education (ISTE), India Member of Institute of Electrical and Electronics Engineers (IEEE), Member of Institute of Electronics and Telecom- munication Engineers (IETE), India and Member of Institute of Engineers, India. His current research includes Information Theory and Coding, Turbo Codes, LDPC codes, Iterative decoding algorithms, wireless sensor network, Mobile Ad Hoc Network, Cognitive Radio.

(iD) https://orcid.org/0000-0002-7798-1359

E-mail: pdapatil@gmail.com

Department of E\&CE

P.D.A. College of Engineering

Kalaburagi, India 